\title{
Neon in carbon nanopores: wetting, growth mechanisms and cluster structures
}

\author{
N.V. Krainyukova \\ B. Verkin Institute for Low Temperature Physics and Engineering of the National Academy of Sciences of Ukraine \\ 47 Lenin Ave., Kharkov 61103, Ukraine \\ Email: ninakrai@yahoo.com \\ krainyukova@ilt.kharkov.ua
}

Received October 20, 2006

\begin{abstract}
Low-temperature high-energy $(50 \mathrm{keV})$ electron diffraction study of size-dependent structures and growth mechanisms of neon samples in multiporous «amorphous» carbon films is presented. Electron diffractograms were analyzed on the basis of the assumption that there exists the cluster size distribution in deposits formed in substrate and multi-shell structures such as icosahedra, decahedra, fcc and hcp were probed for different sizes up to approximately $3 \cdot 10^{4}$ atoms. The analysis was based on the comparison of precise experimental and calculated diffracted intensities with the help of the $R$ (reliability) — factor minimization procedure. Highly reproducible discrete distribution functions of sizes and structures were found. The time-dependent evolution of diffractograms at earlier stages of growth was revealed. Initially distinct diffraction peaks gradually «disappeared» although the total electron beam absorption evidenced that deposited neon was preserved in the porous substrate. We ascribed this effect to diffusion-like gas penetration from larger to smaller pores which resulted in a highly dispersed or even disordered substance. Evidently, clusters initially grown during deposition were later soaked by a sponge-like substrate due to capillary forces.
\end{abstract}

PACS: 61.14. $-\mathbf{x}$ Electron diffraction and scattering;

61.46.-w Nanoscale materials;

67.70.+n Films (including physical adsorption);

81.05.Rm Porous materials; granular materials;

81.05.Uw Carbon, diamond, graphite.

Keywords: carbon nanopores, electron diffraction.

\section{Introduction}

According to the Stranski-Krastanov growth scheme, which was expected to be operative for the noble gas cluster formation on a flat substrate [1], an intermediate layer between the surface and clusters forms first and then noble gas islands grow above. This growth mode owes to the stronger atomic interaction between noble gas atoms and the substrate compared to the interaction between adsorbate atoms themselves. Although theoretical studies of relevant growing processes demonstrated a reduced wetting [2] of adsorbate layers and no equilibrium between the intermediate layer and the islands [3], the electron microscopy observation [4] of the time delay for the cluster growth on graphite was ascribed to the intermediate layer formation.
In the field of nanostructural carbon-based materials with drastically different structures and properties, which is rapidly developing during the last two decades, a variety of growth schemes can be presumed with numerous opportunities of applications. The so-called schwartzites $[5,6]$ formed by negatively curved graphitic sheets are permitted by interconnected channels and can be considered as a porous matrix, which may be filled with different substances under certain conditions. Clusters grown inside pores obey the growth mechanisms typical of confined geometry and demonstrate the absolute prevalence of structures with the five-fold symmetry [7-9]. Such structures were earlier observed in essentially different experiments [7-21] with the only one common feature, i.e., the limited number of interacting atoms during growth. 


\section{Experimental details and analysis method}

The so-called «amorphous» [5] carbon films filled with solidified neon by gas deposition onto the substrate cooled down to liquid helium temperature were studied in high-energy $(50 \mathrm{keV})$ electron diffraction experiments. Neon was precooled to a liquid nitrogen temperature before deposition and was studied at $6 \mathrm{~K}$. During deposition, carbon films were evacuated from backside inside the electron diffraction column to facilitate gas penetration into substrate. The effective thickness $\delta$ of deposits characterized the total amount of condensed materials and the pore filling. All diffraction data were collected in the form of numerical files.

Porous carbon films were produced from vacuum sublimated graphite (by means of the electric current heating of thin carbon rods of approximately $1 \mathrm{~mm}$ or thinner in diameters, the arc discharge avoided) by deposition onto a cleaved $\mathrm{NaCl}$ single crystal surface. Salt sublayers were dissolved in water; carbon films were put onto the copper mesh (with a high-thermal conductivity and transparent for electrons) by means of the floating of carbon films. Prepared in such way, the carbon films were evacuated during several hours (or even days) before gas deposition. Basically the applied procedure was typical of the preparation of «amorphous» carbon films, which are widely used in electron microscopy and diffraction as supporting substrates mainly because of the absence of distinct diffraction peaks from such films superimposed on lines of the material deposited upon substrates. However, it was noticed $[5,8,9]$ that carbon films prepared in such a way also exhibit several important features, which characterize them as a porous matrix with very fine channels. One of them is the absence of the (broad) peak corresponding to distances between basal planes of graphite [8] (see also Fig. 1) while other characteristic peaks were observed and evidenced (allowing for the radial distribution functions $[5,8]$ ) in favor of negatively curved graphitic sheets forming the porous matrix. Several independent observations confirmed the essential difference of such substrates from (e.g.) polycrystalline aluminum films. Simultaneously deposited gases onto these two substrates formed samples, which demonstrated (as compared with each other) the diffraction traces with low for the Al substrate and high for carbon films backgrounds, with thin and distinct peaks on $\mathrm{Al}$ and wide lines onto carbon, in numerous experiments deposits on carbon noticeably evolved with time. These differences increase at higher temperatures and for lighter gases, for instance it was rather difficult to observe distinct diffraction peaks from the hydrogens deposited onto carbon films (in particular, because these peaks gradually disappeared) while on polycrystalline Al films all peaks were distinctly observable.

Diffractograms (Fig. 1) were analyzed using simulations, which were made in a similar way as described pre-

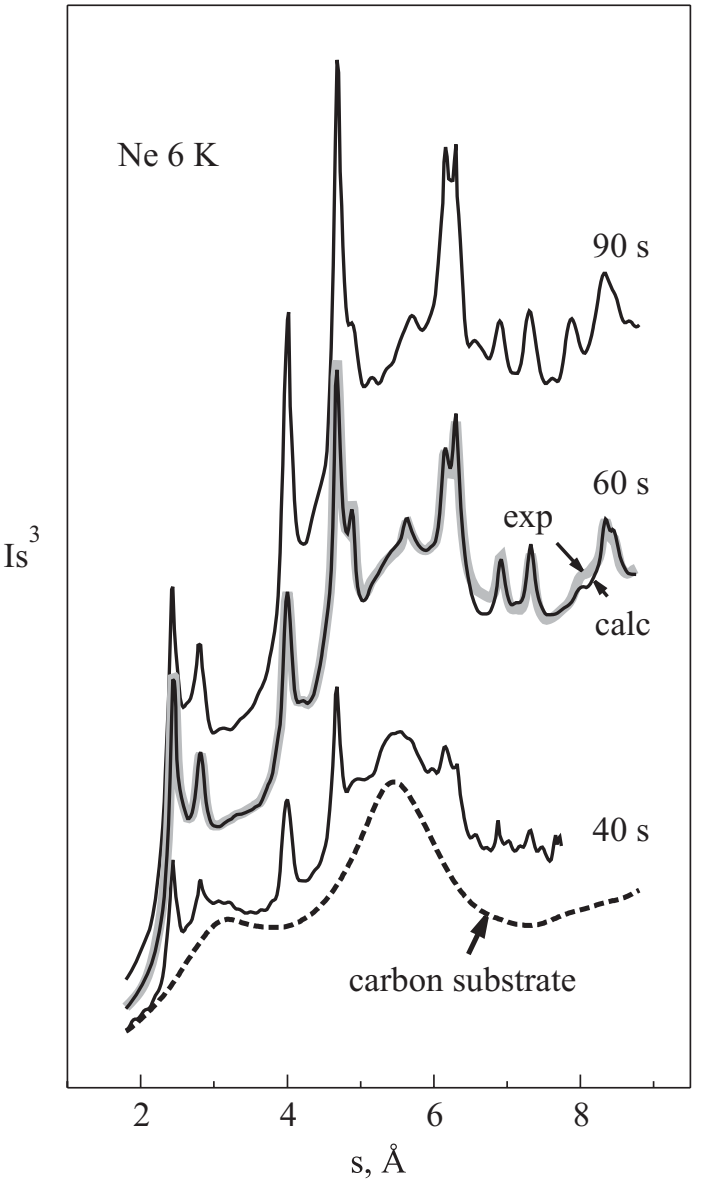

Fig. 1. Experimental diffractograms from $\mathrm{Ne}$ samples recorded for different deposition time intervals $t_{\mathrm{dep}}$. Closest traces are separated from each other by black and gray colors. The experimental diffractogram (exp) for $t_{\text {dep }}=60 \mathrm{~s}$ is superimposed with the calculated one (calc). The diffractogram (lowest) from the carbon film is shown.

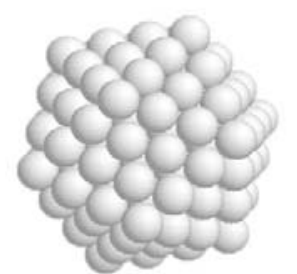

ico

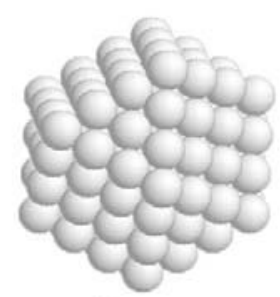

fcc

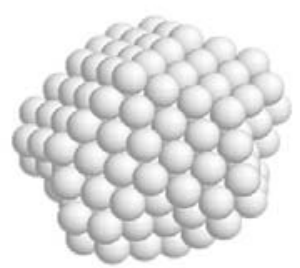

dec

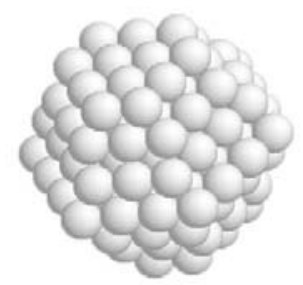

hcp
Fig. 2. Examples of model-clusters used in the simulations of diffraction patterns: icosahedra (ico), decahedra (dec), fcc and hep clusters. 
viously [7-9], i.e., on the basis of the assumption of a wide variety of possible structures and sizes of clusters (Fig. 2). We included in our analysis single crystals such as cube-octahedra and hep clusters as well as multiply twinned particles (MTPs) with five-fold symmetry, i.e., noncrystallographic multishell icosahedra (ico) [22] and Mark's decahedra (dec) [23], with initially from one up to 20 shells for every structural type. The diffracted intensity (per atom) for each cluster $k$ comprising $N_{k}$ atoms was calculated using the Debye formulas:

$$
\begin{aligned}
& I_{\text {calc }, k}(s)=\frac{f^{2}+s^{-4} S(s)}{1-t}+ \\
& +\frac{2 f^{2}}{N_{k}} \exp \left(-\left\langle u^{2}\right\rangle_{k} S^{2}\right)\left(\sum \frac{\sin s r_{i j}}{s r_{i j}}\right)_{k} .
\end{aligned}
$$

Here the fraction $t$ of atoms was assumed not to be bonded to the cluster. The summation in the interference function runs in the cluster $k$ over all pairs of atoms $i>j$, separated by the distance $r_{i j}$; the Debye-Waller factor $\exp \left(-\left\langle u^{2}\right\rangle_{k} S^{2}\right)$ allows for mean-square atomic displacements $\left\langle u^{2}\right\rangle_{k} ; f(s)$ is the elastic atomic scattering factor for electrons; $S(s)$ is the incoherent $\mathrm{x}$-ray scattering factor. The superposition of diffracted intensities $I_{\text {calc }}$ from different clusters $k$ with their relative weights $w_{k}$, i.e., $I_{\text {calc }}=\sum w_{k} I_{\text {calc }, k}$, was compared with the experimental intensities $I_{\exp }$ using the reliability factor $R$ in the form $R=$ $=\Sigma\left|I_{\exp }-I_{\text {calc }}\right| / \Sigma\left(I_{\text {exp }}+I_{\text {calc }}\right)$, where the summation was taken over approximately $300-400$ points along the experimental and calculated diffraction patterns with the equidistant step of $\sim 0.015 \AA^{-1}$ in $s=4 \pi \sin (\theta) / \lambda$ ( $\theta$ is the diffraction angle and $\lambda$ is the wavelength of electrons). The values $w_{k}$ were involved in the total analysis as nearly independent variables [7-9]. The total $\sum w_{k}$ of all relative weights is equal to 1 .

\section{Results and discussion}

Neon gas was condensed on substrate by small portions and diffractograms (Fig. 1) were recorded after every next portion deposited. The carbon substrate contribution was measured before deposition and accounted for in the analysis. The relevant distribution functions over structures and sizes are shown in Fig. 3. They demonstrate a highly reproducible discrete character, which was nearly independent of the effective deposit thickness and implies the self-selection in sizes and structures that may characterize the porous matrix. Clusters with the five-fold symmetry (MTPs) prevail and basically appear in the size intervals predicted in the theory $[11,24]$, i.e., icosahedra for small sizes while decahedra for largest formations. Only three typical sizes were observed and can be ascribed to micro- and mesopores according to the classification [6] because in the wide class
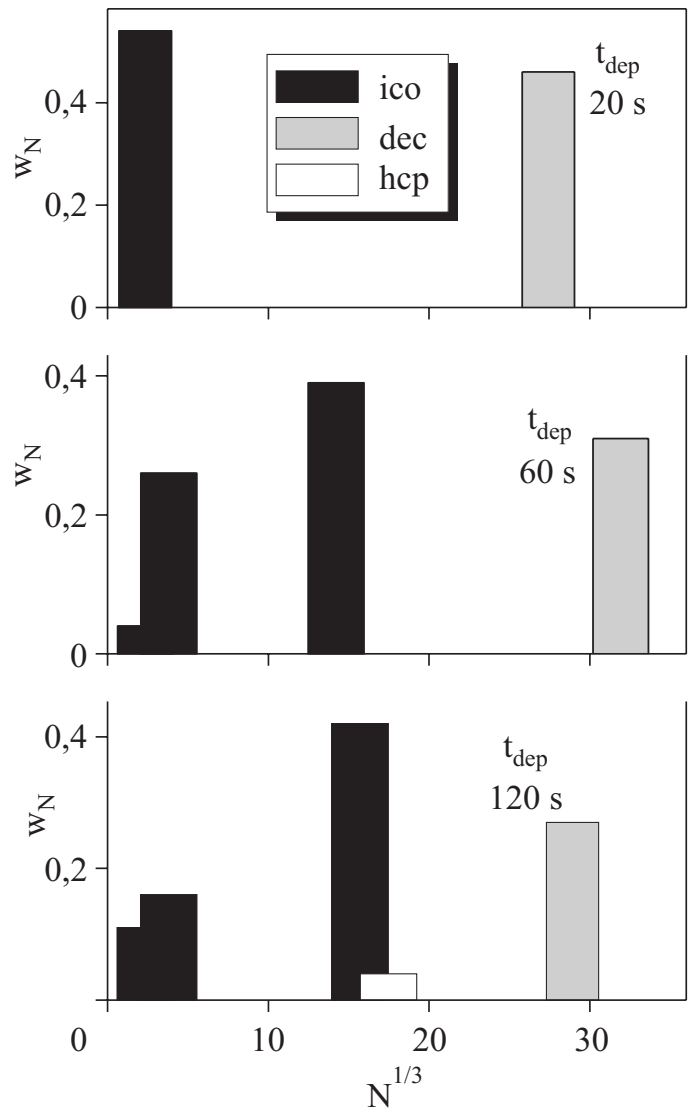

Fig. 3. Distribution functions of Ne clusters over sizes and structures found on the basis of the analysis of experimental diffractograms. The inset shows the structure identifications.

of nanostructured carbon-based materials carbon sheet curvatures and structural arrangements range from nanometers up to several hundred nanometers. The smallest cluster size (about 13-55 atoms) was earlier ascribed [9] to some characteristic structural motif, which forms inside carbon films under the preparation conditions and is preserved even under the film annealing. The next in size pores presumably belong to some kind of large defects $(\sim 40-50 \AA)$ inside films while the largest clusters evidently grow above substrate in essentially open geometry that makes it possible to use these films as supporting substrates in numerous electron microscopy and electron diffraction experiments. Several experimental facts described below confirm our classification.

At earlier growth stages we observed only two types of sizes (Fig. 3), namely, the smallest and the largest ones as well as a time evolution of the diffraction patterns and relevant distributions (Fig. 4). The contribution of the largest clusters rapidly decreases in a few minutes by several percents while the number of atoms in smallest clusters grows up. We conclude that the substance directly deposited on substrate in the form of large clusters later penetrate in smaller pores due to diffusion-like processes. Absence of intermediate sizes in this stage confirms our 


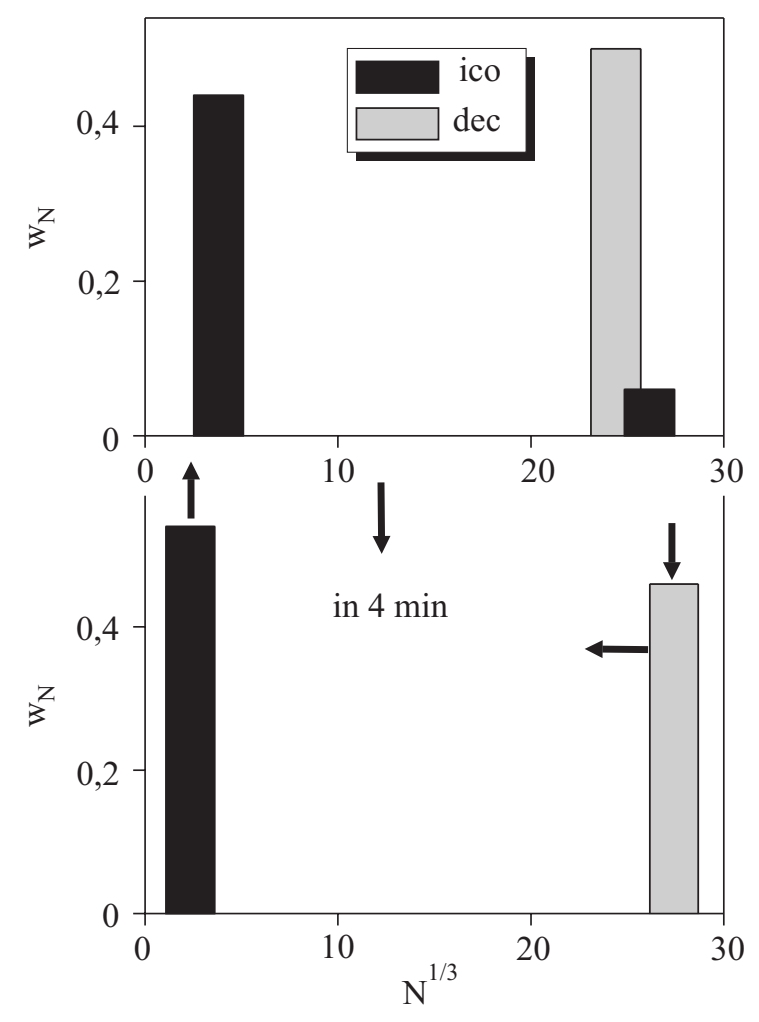

Fig. 4. Time evolution of distribution functions at earlier stages of the film growth: $\delta=15 \AA, t_{\mathrm{dep}}=20 \mathrm{~s}$.

previous assumption concerning large defects inside films because otherwise all sizes would be visible at the beginning. Moreover, smaller sizes also decrease, possibly due to cluster splitting into smaller formations owing to the interaction through pore walls. As we will see below, with growing amount of the deposited material a highly disordered matter forms, which does not belong to any clusters. This observation implies that smaller voids can form inside substrate and are filled at later growth stages with deposited atoms because the potential barriers delay penetration into these positions. Really, our method allows the description not only of the clusters contribution into the resulting diffraction pattern but also the estimation of the total amount $t$ (see the formula given in the previous section) of the part of atoms not bonded with clusters. In Fig. 5 one can see the evolution of this component depending on the effective thickness of the deposited material. We express here the effective thickness by means of the ratio of the total amount of deposited neon atoms $N_{\mathrm{Ne}}$ to the total number of carbon atoms $N_{\mathrm{C}}$ participating in the scattering process, with allowance for their different scattering abilities. In contrast with the anticipated Stranski-Krastanov growth scheme, which presumes the disordered layer formation before the cluster growth, we see no contribution from the atoms not bonded with clusters at earlier growth stages, i.e., clusters grow first. The roughness of inner walls in porous materi-

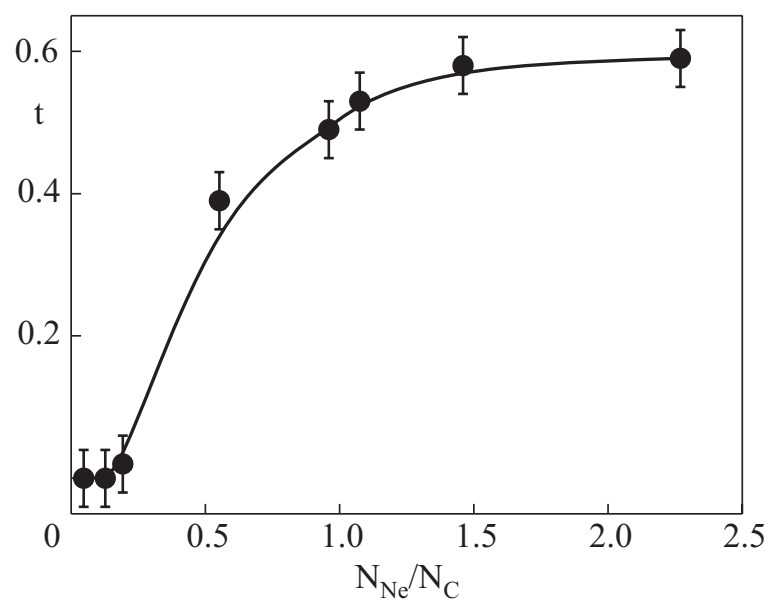

Fig. 5. Dependence of the disordered fraction $t$ on the film filling.

als was presumed [25] to be the reason, which may reduce wetting because of bending energy «penalty» in adsorbate layers picked up by wall roughness. But at the later stages the disordered component appears and increases with filling. Hence, our previous conclusion concerning the diffusion- like penetration of atoms into ever smaller voids is basically the most reasonable. In addition, we can emphasize also the possible role of the «effective pressure» arising in larger pores and «pushing» atoms in the smaller ones.

\section{Summary}

According to the Stranski-Krastanov growth scheme, noble gases deposited on substrates first form an intermediate disordered layer between the substrate and the crystalline phase, which later grows above this layer. Our work does not support this scenario in application to the porous carbon substrate. The crystalline phase appears first in the form of clusters, then due to the diffusion processes the smaller clusters grow inside substrate pores and at last the disordered component appears at latter growth stages, filling the smallest voids inside substrate in positions, where no crystalline phase can exist.

The distribution functions in cluster structures and sizes calculated on the basis of the diffraction pattern analysis demonstrate the unique similarity; they are nearly independent of the deposit effective thickness and presumably characterize the porous carbon matrix. Thus, in line with our previous observations [7-9], the discrete character of micro- and mesopores presumably induces the found self-selection in cluster sizes. The distribution functions exhibit for all objects under study absolute prevalence of MTPs, i.e., clusters with the five-fold symmetry. Comparison with the energy calculations made for different structures showed that both ico and dec clusters were basically found in the size intervals predicted by theory. 
The highly reproducible discrete distribution functions characterize our method as a good application to the pore medium description. Finally we should conclude that the method suggested in this and our previous similar works [7-9] can also be used in the analysis of a wide group of objects, which obey the growth mechanisms typical of confined geometry.

The author is grateful to V.G. Manzhelii, M.A. Strzhemechny, V.V. Khmelenko, V.A. Apkarian, J.-P. Galaup, and V. Kiryukhin for fruitful discussion of the problem, she is also greatly indebted to B.W. van de Waal for numerous valuable comments and also for the software provided for cluster modelling.

1. J.A. Venables, G.D.T. Spiller, and M. Hanbücken, Rep. Progr. Phys. 47, 399 (1984).

2. F.T. Gittes and M. Schick, Phys. Rev. B30, 209 (1984).

3. J.E. Prieto and I. Markov, Phys. Rev. B66, 073408 (2002).

4. J.A. Venables and D.J. Ball, Proc. Roy. Soc. (London) A322, 331 (1971).

5. S.J. Townsend, T.J. Lenosky, D.A. Muller, C.S. Nickols, and V. Elser, Phys. Rev. Lett. 69, 921 (1992).

6. E. Barborini, P. Piseri, P. Milani, G. Benedek, C. Ducati, and J. Robertson, Appl. Phys. Lett. 81, 3359 (2002).

7. N.V. Krainyukova and B.W. van de Waal, Thin Solid Films 459, 169 (2004)

8. N.V. Krainyukova, Surf. Interface Anal. 38, 469 (2006).

9. N.V. Krainyukova, Thin Solid Films 515, 2792 (2006).

10. H. Hofmeister, in: Encyclopedia of Nanoscience and Nanotechnology, H.S. Nalwa (ed.), V. 3, American Scientific Publishers, Los Angeles (2004).
11. B. Raoult, J. Farges, M.F. De Feraudy, and G. Torchet, Philos. Mag. B60, 881 (1989).

12. B.W. van de Waal, The FCC/HCP Dilemma, B.V. Febodruk (ed.), Enschede (1997).

13. B.W. van de Waal, G. Torchet, and M.-F. de Feraudy, Chem. Phys. Lett. 331, 57 (2000).

14. T. Ikeshoji, G. Torchet, M.-F. de Feraudy, and K. Koga, Phys. Rev. E63, 031101 (2001).

15. O.G. Danylchenko, S.I. Kovalenko, and V.N. Samovarov, Fiz. Nizk. Temp. 30, 986 (2004) [Low Temp. Phys. 30, 743 (2004)].

16. O.G. Danylchenko, S.I. Kovalenko, and V.N. Samovarov, Fiz. Nizk. Temp. 30, 226 (2004) [Low Temp. Phys. 30, 166 (2004)].

17. D. Reinhard, B.D. Hall, D. Ugarte, and R. Monot, Phys. Rev. B55, 7868 (1997).

18. D. Reinhard, B.D. Hall, P. Berthoud, S. Valkealahti, and R. Monot, Phys. Rev. B58, 4917 (1998).

19. V. Kiryukhin, B. Keimer, R.E. Boltnev, V.V. Khmelenko, and E.B. Gordon, Phys. Rev. Lett. 79, 1774 (1997).

20. S.I. Kiselev, V.V. Khmelenko, D.M. Lee, V. Kiryukhin, R.E. Boltnev, E.B. Gordon, and B. Keimer, Phys. Rev. B65, 024517 (2001).

21. E.P. Bernard, R.E. Boltnev, V.V. Khmelenko, V. Kiryukhin, S.I. Kiselev, and D.M. Lee, Phys. Rev. B69, 104201 (2004).

22. A.L. Mackay, Acta Cryst. 15, 916 (1962).

23. L.D. Marks, Philos. Mag. A49, 81 (1984).

24. N.V. Krainyukova, Thin Solid Films 515, 1658 (2006).

25. A. Esztermann, M. Heni, H. Löwen, J. Klier, M. Sohaili, and P. Leiderer, Phys. Rev. Lett. 88, 055702 (2002). 